\title{
Heat Tolerance and Insect, Diseases Reaction in Eggplant Hybrids during the Summer Season in Bangladesh
}

\author{
Akm Quamruzzaman ${ }^{*}$, M. Nazim Uddin ${ }^{1}$, Ferdouse Islam ${ }^{1}$ and M. A. Z. Chowdhury ${ }^{2}$ \\ ${ }^{1}$ Olericulture Division, Horticulture Research Centre, Bangladesh Agricultural Research Institute, Gazipur, \\ Bangladesh \\ ${ }^{2}$ Bangladesh Agricultural Research Council, Farmgate, Dhaka-1207, Bangladesh \\ Email: akmqzs@gmail.com
}

\begin{abstract}
A study was evaluated with 20 Fi's lines/ variety at Olericulture Division, Horticulture Research Centre (HRC), Bangladesh Agricultural Research Institute, Gazipur during the summer season of 2017 to develop new high yielding hybrids having tolerance to high temperature and insect [eggplant fruit and shoot borer (EFSB)], disease [bacterial wilt and phomopsis blight]. The concern lines/ variety varied significantly for their response to all characters $(\mathrm{P}<0.05)$. The hybrid $\mathrm{F}_{1} 14 \mathrm{X} 216$ required minimum 90.33 days to first harvest. Highest marketable fruit number was found by $\mathrm{F}_{1} 14 \mathrm{x}$ 233 (66.92). Heavier sized fruit was obtained by $\mathrm{F}_{1} 19 \mathrm{X} 598.11 \mathrm{~g}$ ), which was identical with $\mathrm{F}_{1} 5 \mathrm{X} 8$ $(95.17 \mathrm{~g}), \mathrm{F}_{1} 5 \mathrm{X} 203(95.17 \mathrm{~g})$. The plant height ranges at first and last harvest was $70.00-97.88$ days and 92.89 - 137.11 days, respectively. The fruit infection by EFSB was $7.40-15.40 \%$, while lowest in $\mathrm{F}_{1} 5 \mathrm{X} 8(7.40 \%)$, which was identical to $\mathrm{F}_{1} 13 \mathrm{X} 11$ (7.45 \%). Zero percent bacterial wilt and phomopsis blight diseases incidence was observed in $\mathrm{F}_{1} 1 \mathrm{X} 216, \mathrm{~F}_{1}$ 1X233, $\mathrm{F}_{1}$ 14X233, $\mathrm{F}_{1}$ 19X203, $\mathrm{F}_{1} 19 \mathrm{X} 216$ in field level. The yield range of eggplant hybrids was 24.68- $41.49 \mathrm{t} / \mathrm{ha}$. The highest fruit yield was produced from $\mathrm{F}_{1}$ 14X233 (41.39 t/ha), followed by $\mathrm{F}_{1}$ 14X216 (35.70 t/ha), F 1X233 (34.08 t/ha), $\mathrm{F}_{1}$ 19X216 (33.80 t/ha), F 5X233 (33.75 t/ha), $\mathrm{F}_{1} 5 \mathrm{X} 8$ (31.54 t/ha), and lowest yield were recorded from $\mathrm{F}_{1} 14 \mathrm{X} 5$ (24.68 t/ha). Though the yield was higher in the hybrids $\mathrm{F}_{1} 14 \mathrm{X} 233, \mathrm{~F}_{1} 14 \mathrm{X} 216, \mathrm{~F}_{1} 1 \mathrm{X} 233, \mathrm{~F}_{1}$ 19X216, F $15 X 233, F_{1} 5 X 8$, but considering attractive fruit shape and fruit colour, earliness, tolerance to high temperature, fruit infection by EFSB, bacterial wilt and phomopsis blight infestation, the hybrids $\mathrm{F}_{1}$ 1X233, $\mathrm{F}_{1}$ 5X8, $\mathrm{F}_{1}$ 13X11, $\mathrm{F}_{1}$ 14X233, $\mathrm{F}_{1}$ 14X216, $\mathrm{F}_{1}$ 19X216, $\mathrm{F}_{1}$ 19X233, $\mathrm{F}_{1}$ 21X221B were found best. So these seven hybrids can be selected for advance trial.
\end{abstract}

Keywords: Heat tolerance, insect and disease reaction, eggplant hybrids, summer season, Bangladesh.

\section{Introduction}

Eggplant (Solanum melongena L.) is the most popular vegetable crop in respect of total acreage $(50,415$ ha) and production (504,817 ton) in Bangladesh with an average yield of 10.0 tons per hectare (Anon., 2017), which is very low as compared to that in other eggplant producing countries. High yielding variety either hybrid and open pollinated $(\mathrm{OP})$ varieties are important factors for maximizing the yield of eggplant. Increased productivity in the shortest time can be achieved through heterosis breeding (Kakizaki, 1931). Highly varied consumer acceptance demands development of high-yielding F1 hybrids. Exploitation of hybrid vigor has become a potential tool for improvement in eggplant (Bavage et al., 2005; Dharwad et al., 2011; Prabhu et al., 2005). The estimation of heterosis for yield and its component characters would be useful in determining the best hybrid combination. So, there is a great chance to get higher yield using hybrid exploitation in eggplant. Eggplant has the potential for improvement through heterosis breeding, which can be further utilized for development of desirable recombinants.

Eggplant offers the possibility of improvement through heterosis breeding and continues to be a choice of breeders for exploitation of heterosis due to the hardy nature of the plant, comparatively large size of flowers, and large numbers of seed produced by a single act of pollination. Depending on the varieties used, resistance to $R$. solanacearum is controlled by a single dominant gene (Ajjappalavara et al., 2008; Cao et al., 2009; Gopinath and Zhu et al., 2004) or one recessive gene (Sun et al., 2008) or a dominant polygene (Chaudhary, 2000) or recessive polygene (Feng et al., 2003). 
A heterosis study was used to determine heterosis over better parent for 12 characters in 2014. Best five crosses were selected on the basis of their per se (mean) performance: heterosis and the SCA effects. These hybrids could be used commercially due to high yield and low percentage disease for bacterial wilt disease and phomopsis blight. In our country, the yield of eggplant during summer season is low compare to winter season. The main causes of low production are scarcity of summer type heat tolerant good quality eggplant hybrid varieties and also scarcity of bacterial wilt and phomopsis blight disease tolerant good quality eggplant hybrid varieties. So keeping this information, this study was undertaken to study the performance of hybrids regarding yield and having tolerance to high temperature and eggplant fruit and shoot borer and bacterial wilt disease and phomopsis blight.

\section{Materials and Methods}

The study was conducted at the experimental farm of Olericulture Division, Horticulture Research Centre (HRC), Bangladesh Agricultural Research Institute, Gazipur during the summer of 2017. The experimental field was at $23.9917^{\circ} \mathrm{N}$ Latitude and $90.4124^{\circ} \mathrm{E}$ Longitudes having an elevation of $8.2 \mathrm{~m}$ from sea level. Twenty eggplant hybrid/ varieties viz., $\mathrm{F}_{1} 1 \mathrm{X} 216, \mathrm{~F}_{1} 1 \mathrm{X} 233, \mathrm{~F}_{1} 3 \mathrm{X} 229, \mathrm{~F}_{1}$ 4X203, $\mathrm{F}_{1} 5 \mathrm{X} 8$, $\mathrm{F}_{1}$ 5X203, $\mathrm{F}_{1}$ 5X233, $\mathrm{F}_{1}$ 13X11, $\mathrm{F}_{1} 14 \mathrm{X} 5, \mathrm{~F}_{1} 14 \mathrm{X} 8, \mathrm{~F}_{1}$ 14X216, $\mathrm{F}_{1}$ 14X233, $\mathrm{F}_{1}$ 18X216, $\mathrm{F}_{1}$ 18X233, $\mathrm{F}_{1}$ 19X5, $\mathrm{F}_{1}$ 19X203, $\mathrm{F}_{1}$ 19X216, $\mathrm{F}_{1}$ 19X233, $\mathrm{F}_{1}$ 21X221B, BARI Hybrid Begun-4were included in the study. The seeds were sown on the seedbed on 15 March 2017. Thirty-two days old seedlings were transplanted in the main field on 16 April, 2017. The experiment was laid out in a RCB design with three replications. The plot size was $7.5 \times 0.70 \mathrm{~m}$ and 10 plants were accommodated in a plot with a plant spacing of 75 $\mathrm{cm}$ apart in single row maintaining a row to row distance of $1 \mathrm{~m}$ with $50 \mathrm{~cm}$ drain. The land was fertilized with cowdung, N, P, K, S, Zn and B @ 10,000 100, 30, 75, 13, 1.5 and $0.8 \mathrm{~kg} / \mathrm{ha}$, respectively. One third of the cow-dung and half of $\mathrm{P}$ and full of $\mathrm{S}, \mathrm{Zn}$ and $\mathrm{B}$ were applied during final land preparation. Rest of cow-dung and $\mathrm{P}$ and $1 / 3$ of $\mathrm{K}$ were applied as basal in pit. Entire amount of $\mathrm{N}$ and rest of $\mathrm{K}$ were applied in five equal installment starting from 20 days after transplanting. Rest four installments were applied at vegetative, flowering, initial fruiting stage and pick fruiting stage. Concern all operations like- irrigation, weeding, crop protection measures and other intercultural operations were done following standard cultural practices. Data were recorded from five randomly selected plants per entry per replication on - days to 1st harvest, marketable fruit number/ plant, average fruit weight (g), fruit weight/ plant $(\mathrm{kg})$, fruit length $(\mathrm{cm})$, fruit diameter $(\mathrm{cm})$, plant height at 1st harvest $(\mathrm{cm})$, plant height at last harvest $(\mathrm{cm})$, fruit infection by EFSB (\%), bacterial wilt infestation (\%), little leaf infestation (\%), phomopsis blight infestation (\%), fruit yield (t/ha), fruit shape and fruit colour. The information on different characters was statistically analyzed.

Table 1. The weather data of Gazipur during summer season, 2017

\begin{tabular}{c|ccc|c}
\hline \multirow{2}{*}{ Month 2017} & \multicolumn{3}{|c|}{ Temperature } & \multirow{2}{*}{ Humidity (\%) } \\
\cline { 2 - 4 } & High & Low & Average & \\
\hline April & $36{ }^{\circ} \mathrm{C}$ & $20{ }^{\circ} \mathrm{C}$ & $28{ }^{\circ} \mathrm{C}$ & 72 \\
May & $36{ }^{\circ} \mathrm{C}$ & $21^{\circ} \mathrm{C}$ & $30{ }^{\circ} \mathrm{C}$ & 71 \\
June & $40{ }^{\circ} \mathrm{C}$ & $24{ }^{\circ} \mathrm{C}$ & $30{ }^{\circ} \mathrm{C}$ & 78 \\
July & $36{ }^{\circ} \mathrm{C}$ & $25^{\circ} \mathrm{C}$ & $29{ }^{\circ} \mathrm{C}$ & 81 \\
Aug & $34{ }^{\circ} \mathrm{C}$ & $26{ }^{\circ} \mathrm{C}$ & $29{ }^{\circ} \mathrm{C}$ & 82 \\
Sep & $35{ }^{\circ} \mathrm{C}$ & $25^{\circ} \mathrm{C}$ & $29{ }^{\circ} \mathrm{C}$ & 81 \\
\hline
\end{tabular}

Table 2. Chemical properties of initial soil of the experimental field of BARI, Gazipur during summer season, 2017

\begin{tabular}{|c|c|c|c|c|c|c|c|c|c|c|c|c|c|}
\hline \multirow[t]{2}{*}{ Locations } & $\mathrm{pH}$ & $\mathrm{OM}$ & $\mathrm{Ca}$ & $\mathrm{Mg}$ & K & \multirow{2}{*}{$\begin{array}{c}\text { Total } \\
\mathrm{N} \%\end{array}$} & $\mathrm{P}$ & $\mathrm{S}$ & $\mathrm{Zn}$ & $\mathrm{B}$ & $\mathrm{Fe}$ & Mn & $\mathrm{Cu}$ \\
\hline & & $\%$ & & q 100 & & & \multicolumn{7}{|c|}{$\mu \mathrm{g} \mathrm{g}^{-1}$} \\
\hline Gazipur & 7.4 & 0.93 & 5.47 & 2.41 & 0.17 & 0.054 & 14.9 & 17.3 & 1.64 & 0.21 & 33.6 & 4.3 & 2.3 \\
\hline Critical level & & & 2.0 & 0.50 & 0.12 & 0.12 & 7 & 10 & 0.6 & 0.20 & 4.0 & 1.0 & 0.2 \\
\hline
\end{tabular}




\section{Results and Discussion}

Mean performances of eggplant hybrids are presented in Table 3, 4, 5 and Figure 1. The hybrids varied significantly $(\mathrm{P}<0.05)$ for their response to days to 1st harvest, marketable fruit number/ plant, average fruit weight, fruit weight/ plant, fruit length, fruit diameter, plant height at 1st harvest, plant height at last harvest, fruit infection by EFSB, bacterial wilt infestation, fruit yield. In respect of days to first harvest, the earliest line was $\mathrm{F}_{1}$ 14X216 (90.33 days) followed by $\mathrm{F}_{1}$ 19X216 (92.60 days), $\mathrm{F}_{1} 4 \mathrm{X} 203$ (93.70 days) and hybrid $\mathrm{F}_{1}$ 1X233was the most delayed (106.00days). The range of marketable fruit number was $(26.56-66.92)$. The highest marketable fruit number per plant was counted in $\mathrm{F}_{1} 14 \mathrm{x} 233$ (66.92) which followed by $\mathrm{F}_{1}$ 14X216 (54.10), $\mathrm{F}_{1}$ 1X233 (47.16), F 1 19X233 (43.78), $\mathrm{F}_{1}$ 19X216 (43.12), while lowest fruit number was counted in $\mathrm{F}_{1} 19 \mathrm{X} 5$ (26.56). Average fruit weight is an important criterion to select a high yielder line. The heaviest fruit was produced in $\mathrm{F}_{1} 19 \mathrm{X} 598.11 \mathrm{~g}$ ), which was identical withF 5 X8 (95.17 g), F 5X203 (95.17 g), while lightest fruit was in $\mathrm{F}_{1}$ 14X233 (55.93 g). Fruit weight/ plant was maximum in $\mathrm{F}_{1} 14 \mathrm{X} 233(3.76 \mathrm{~kg})$ followed by $\mathrm{F}_{1} 14 \mathrm{X} 216(3.24 \mathrm{~kg}), \mathrm{F}_{1} 19 \mathrm{X} 216(3.07 \mathrm{~kg}), \mathrm{F}_{1}$ 1X233 (3.09 kg), F 5 X233 (3.00 kg), F 5 X8 $(2.87 \mathrm{~kg})$, while minimum was in $\mathrm{F}_{1} 14 \mathrm{X} 5(2.24 \mathrm{~kg})$. Identical longest fruit was produced by $\mathrm{F}_{1} 1 \times 233(19.10 \mathrm{~cm})$ and $\mathrm{F}_{1} 19 \mathrm{X} 233(18.72 \mathrm{~cm})$ followed by $\mathrm{F}_{1}$ $1 \times 216(17.20 \mathrm{~cm})$ and $F_{1} 14 X 5$ produced the shortest fruit $(8.57 \mathrm{~cm})$. Maximum diameter fruit was produced by the line $\left.\mathrm{F}_{1} 5 \mathrm{X} 86.75 \mathrm{~cm}\right)$ followed by $\mathrm{F}_{1} 21 \mathrm{X} 221 \mathrm{~B}(5.62 \mathrm{~cm})$, BARI Hybrid Begun 4 (5.42 $\mathrm{cm}), \mathrm{F}_{1} 18 \mathrm{X} 216(5.15 \mathrm{~cm})$ and minimum was by $\mathrm{F}_{1} 14 \mathrm{X} 233(2.95 \mathrm{~cm})$. The range of plant height at first and last harvest was 70.00 - 97.88 days and 92.89 - 137.11 days, respectively.

Table 3. The yield and yield contributing characters of 20 hybrids/ variety of eggplant

\begin{tabular}{|c|c|c|c|c|}
\hline Treatment & $\begin{array}{c}\text { Days to } 1 \text { st } \\
\text { harvest }\end{array}$ & $\begin{array}{c}\text { Marketable fruit } \\
\text { number/plant }\end{array}$ & $\begin{array}{c}\text { Average fruit } \\
\text { weight }(\mathrm{g})\end{array}$ & $\begin{array}{c}\text { Fruit weight/ } \\
\text { plant }(\mathrm{kg})\end{array}$ \\
\hline $\mathrm{F}_{1} 1 \mathrm{X} 216$ & $103.45 \mathrm{~b}$ & 39.60 c-e & $69.80 \mathrm{~g}$ & $2.76 \mathrm{~b}-\mathrm{e}$ \\
\hline $\mathrm{F}_{1} 1 \mathrm{X} 233$ & $106.00 \mathrm{a}$ & $47.16 \mathrm{bc}$ & $66.20 \mathrm{~h}$ & $3.09 \mathrm{bc}$ \\
\hline $\mathrm{F}_{1} 3 \mathrm{X} 229$ & $96.80 \mathrm{f}-\mathrm{h}$ & $32.853 \mathrm{e}-\mathrm{i}$ & 74.33 ef & $2.43 \mathrm{de}$ \\
\hline $\mathrm{F}_{1} 4 \mathrm{X} 203$ & $93.70 \mathrm{ij}$ & 32.18 e-i & $72.50 \mathrm{fg}$ & $2.32 \mathrm{e}$ \\
\hline $\mathrm{F}_{1} 5 \mathrm{X} 8$ & $103.07 \mathrm{bc}$ & $30.23 \mathrm{f}-\mathrm{i}$ & $95.17 \mathrm{a}$ & $2.87 \mathrm{~b}-\mathrm{e}$ \\
\hline $\mathrm{F}_{1} 5 \mathrm{X} 203$ & $96.60 \mathrm{hi}$ & 27.74 g-i & $95.17 \mathrm{a}$ & $2.60 \mathrm{~b}-\mathrm{e}$ \\
\hline $\mathrm{F}_{1} 5 \mathrm{X} 233$ & $97.40 \mathrm{f}-\mathrm{h}$ & $38.38 \mathrm{~d}-\mathrm{f}$ & $79.88 \mathrm{~d}$ & $3.00 \mathrm{~b}-\mathrm{d}$ \\
\hline $\mathrm{F}_{1} 13 \mathrm{X} 11$ & $99.67 \mathrm{~d}-\mathrm{f}$ & $30.90 \mathrm{f}-\mathrm{i}$ & $83.40 \mathrm{c}$ & $2.56 \mathrm{c}-\mathrm{e}$ \\
\hline $\mathrm{F}_{1} 14 \mathrm{X} 5$ & $104.13 \mathrm{ab}$ & $29.45 \mathrm{~g}-\mathrm{i}$ & $76.31 \mathrm{e}$ & $2.24 \mathrm{e}$ \\
\hline $\mathrm{F}_{1} 14 \mathrm{X} 8$ & $103.45 \mathrm{~b}$ & $38.00 \mathrm{~d}-\mathrm{f}$ & $71.27 \mathrm{fg}$ & $2.70 \mathrm{~b}-\mathrm{e}$ \\
\hline $\mathrm{F}_{1} 14 \mathrm{X} 216$ & $90.33 \mathrm{k}$ & $54.10 \mathrm{~b}$ & $59.97 \mathrm{i}$ & $3.24 \mathrm{ab}$ \\
\hline $\mathrm{F}_{1} 14 \mathrm{X} 233$ & $101.67 \mathrm{~b}-\mathrm{d}$ & $66.92 \mathrm{a}$ & $55.93 \mathrm{j}$ & $3.76 \mathrm{a}$ \\
\hline $\mathrm{F}_{1} 18 \mathrm{X} 216$ & 99.60 d-g & 27.16 hi & $88.40 \mathrm{~b}$ & $2.38 \mathrm{e}$ \\
\hline $\mathrm{F}_{1} 18 \mathrm{X} 233$ & $102.73 \mathrm{bc}$ & $31.56 \mathrm{e}-\mathrm{i}$ & $80.52 \mathrm{~cd}$ & $2.53 \mathrm{c}-\mathrm{e}$ \\
\hline $\mathrm{F}_{1} 19 \mathrm{X} 5$ & $100.45 \mathrm{c}-\mathrm{e}$ & $26.56 \mathrm{i}$ & $98.11 \mathrm{a}$ & $2.60 \mathrm{~b}-\mathrm{e}$ \\
\hline $\mathrm{F}_{1} 19 \times 203$ & $97.73 \mathrm{e}-\mathrm{h}$ & $35.67 \mathrm{~d}-\mathrm{h}$ & $71.27 \mathrm{fg}$ & $2.54 \mathrm{c}-\mathrm{e}$ \\
\hline $\mathrm{F}_{1} 19 \mathrm{X} 216$ & $92.60 \mathrm{jk}$ & $43.12 \mathrm{~cd}$ & $71.27 \mathrm{fg}$ & $3.07 \mathrm{~b}-\mathrm{d}$ \\
\hline $\mathrm{F}_{1} 19 \mathrm{X} 233$ & $96.67 \mathrm{~g}-\mathrm{i}$ & $43.78 \mathrm{~cd}$ & $63.40 \mathrm{~h}$ & $2.77 \mathrm{~b}-\mathrm{e}$ \\
\hline $\mathrm{F}_{1} 21 \mathrm{X} 221 \mathrm{~B}$ & $98.33 \mathrm{e}-\mathrm{h}$ & $31.36 \mathrm{e}-\mathrm{i}$ & $81.57 \mathrm{~cd}$ & $2.53 \mathrm{c}-\mathrm{e}$ \\
\hline BARI Hybrid Begun 4 & $103.11 \mathrm{bc}$ & $35.98 \mathrm{~d}-\mathrm{g}$ & $71.93 \mathrm{fg}$ & $2.59 \mathrm{c}-\mathrm{e}$ \\
\hline Level of sig. & $*$ & $*$ & $*$ & $*$ \\
\hline $\mathrm{CV}(\%)$ & 1.82 & 13.93 & 2.45 & 14.41 \\
\hline
\end{tabular}


Table 3. Contd.

\begin{tabular}{|c|c|c|c|c|}
\hline Treatment & $\begin{array}{l}\text { Fruit length } \\
(\mathrm{cm})\end{array}$ & $\begin{array}{l}\text { Fruit diameter } \\
(\mathrm{cm})\end{array}$ & $\begin{array}{c}\text { Plant height at 1st } \\
\text { harvest }(\mathrm{cm})\end{array}$ & $\begin{array}{c}\text { Plant height at last } \\
\text { harvest }(\mathrm{cm})\end{array}$ \\
\hline $\mathrm{F}_{1} 1 \mathrm{X} 216$ & $17.20 \mathrm{~b}$ & $2.65 \mathrm{l}$ & $80.00 \mathrm{i}$ & $121.89 \mathrm{a}-\mathrm{c}$ \\
\hline $\mathrm{F}_{1} 1 \mathrm{X} 233$ & $19.10 \mathrm{a}$ & $3.59 \mathrm{jk}$ & $97.88 \mathrm{a}$ & $137.11 \mathrm{a}$ \\
\hline $\mathrm{F}_{1} 3 \mathrm{X} 229$ & $11.43 \mathrm{f}$ & $3.35 \mathrm{k}$ & $83.00 \mathrm{~g}$ & $127.55 \mathrm{ab}$ \\
\hline $\mathrm{F}_{1} 4 \mathrm{X} 203$ & $9.23 \mathrm{hi}$ & $5.00 \mathrm{de}$ & $76.33 \mathrm{k}$ & $110.00 \mathrm{~b}-\mathrm{d}$ \\
\hline $\mathrm{F}_{1} 5 \mathrm{X} 8$ & $13.55 \mathrm{~d}$ & $6.75 \mathrm{a}$ & $91.22 \mathrm{~b}$ & $118.78 \mathrm{a}-\mathrm{c}$ \\
\hline $\mathrm{F}_{1} 5 \mathrm{X} 203$ & $8.75 \mathrm{jk}$ & $4.65 \mathrm{fg}$ & $76.33 \mathrm{k}$ & $110.00 \mathrm{~b}-\mathrm{d}$ \\
\hline $\mathrm{F}_{1} 5 \mathrm{X} 233$ & $9.07 \mathrm{ij}$ & $4.09 \mathrm{i}$ & $87.66 \mathrm{~d}$ & $118.45 \mathrm{a}-\mathrm{c}$ \\
\hline $\mathrm{F}_{1} 13 \mathrm{X} 11$ & $8.81 \mathrm{jk}$ & $3.69 \mathrm{j}$ & $78.00 \mathrm{j}$ & $114.11 \mathrm{~b}-\mathrm{d}$ \\
\hline $\mathrm{F}_{1} 14 \mathrm{X} 5$ & $8.57 \mathrm{k}$ & $4.74 \mathrm{e}-\mathrm{g}$ & $78.00 \mathrm{j}$ & $117.11 \mathrm{a}-\mathrm{c}$ \\
\hline $\mathrm{F}_{1} 14 \mathrm{X} 8$ & $15.48 \mathrm{c}$ & $3.35 \mathrm{k}$ & $80.00 \mathrm{i}$ & $117.45 \mathrm{a}-\mathrm{c}$ \\
\hline $\mathrm{F}_{1} 14 \mathrm{X} 216$ & $13.37 \mathrm{~d}$ & $3.35 \mathrm{k}$ & $81.00 \mathrm{~h}$ & $117.78 \mathrm{a}-\mathrm{c}$ \\
\hline $\mathrm{F}_{1} 14 \mathrm{X} 233$ & $10.70 \mathrm{~g}$ & $2.95 \mathrm{l}$ & $70.33 \mathrm{~m}$ & $102.22 \mathrm{~cd}$ \\
\hline $\mathrm{F}_{1} 18 \mathrm{X} 216$ & $11.61 \mathrm{f}$ & $5.15 \mathrm{~cd}$ & $89.66 \mathrm{c}$ & $92.89 \mathrm{~d}$ \\
\hline $\mathrm{F}_{1} 18 \mathrm{X} 233$ & $9.53 \mathrm{~h}$ & $4.86 \mathrm{~d}-\mathrm{f}$ & $85.66 \mathrm{e}$ & $119.78 \mathrm{a}-\mathrm{c}$ \\
\hline $\mathrm{F}_{1} 19 \mathrm{X} 5$ & $12.57 \mathrm{e}$ & $4.55 \mathrm{gh}$ & $80.00 \mathrm{i}$ & $120.11 \mathrm{a}-\mathrm{c}$ \\
\hline $\mathrm{F}_{1} 19 \mathrm{X} 203$ & $10.70 \mathrm{~g}$ & $4.98 \mathrm{de}$ & 73.331 & $112.00 \mathrm{~b}-\mathrm{d}$ \\
\hline $\mathrm{F}_{1} 19 \mathrm{X} 216$ & $15.27 \mathrm{c}$ & $4.32 \mathrm{hi}$ & $83.00 \mathrm{~g}$ & $125.55 \mathrm{ab}$ \\
\hline $\mathrm{F}_{1} 19 \mathrm{X} 233$ & $18.72 \mathrm{a}$ & 2.851 & $83.00 \mathrm{~g}$ & $114.45 \mathrm{~b}-\mathrm{d}$ \\
\hline $\mathrm{F}_{1} 21 \mathrm{X} 221 \mathrm{~B}$ & $11.00 \mathrm{~g}$ & $5.62 \mathrm{~b}$ & $70.00 \mathrm{~m}$ & $111.78 \mathrm{~b}-\mathrm{d}$ \\
\hline BARI Hybrid Begun 4 & $9.00 \mathrm{ij}$ & $5.42 \mathrm{bc}$ & $84.00 \mathrm{f}$ & $131.22 \mathrm{ab}$ \\
\hline Level of sig. & $*$ & $*$ & $*$ & $*$ \\
\hline $\mathrm{CV}(\%)$ & 1.92 & 4.34 & 0.58 & 11.24 \\
\hline
\end{tabular}

Table 4. Fruit infection by EFSB, infestation by bacterial wilt infestation and phomopsis blight of 20 hybrids/ variety of eggplant

\begin{tabular}{|c|c|c|c|}
\hline Treatment & $\begin{array}{c}\text { Fruit infection by } \\
\text { EFSB (\%) }\end{array}$ & $\begin{array}{c}\text { Bacterial wilt } \\
\text { infestation (\%) }\end{array}$ & $\begin{array}{c}\text { Phomopsis blight } \\
\text { infestation }(\%)\end{array}$ \\
\hline $\mathrm{F}_{1} 1 \mathrm{X} 216$ & $9.40 \mathrm{f}$ & $0.00 \mathrm{c}$ & $0.00 \mathrm{~b}$ \\
\hline $\mathrm{F}_{1} 1 \mathrm{X} 233$ & $12.40 \mathrm{c}$ & $0.00 \mathrm{c}$ & $0.00 \mathrm{~b}$ \\
\hline $\mathrm{F}_{1} 3 \mathrm{X} 229$ & $10.40 \mathrm{e}$ & $10.00 \mathrm{ab}$ & $10.00 \mathrm{a}$ \\
\hline $\mathrm{F}_{1} 4 \mathrm{X} 203$ & $11.40 \mathrm{~d}$ & $3.33 \mathrm{bc}$ & $0.00 \mathrm{~b}$ \\
\hline $\mathrm{F}_{1} 5 \mathrm{X} 8$ & $7.40 \mathrm{~h}$ & $3.33 \mathrm{bc}$ & $0.00 \mathrm{~b}$ \\
\hline $\mathrm{F}_{1} 5 \mathrm{X} 203$ & $15.40 \mathrm{a}$ & $13.33 \mathrm{a}$ & $0.00 \mathrm{~b}$ \\
\hline $\mathrm{F}_{1} 5 \mathrm{X} 233$ & $9.60 \mathrm{f}$ & $0.00 \mathrm{c}$ & $3.33 \mathrm{ab}$ \\
\hline $\mathrm{F}_{1} 13 \mathrm{X} 11$ & $7.45 \mathrm{~h}$ & $3.33 \mathrm{bc}$ & $0.00 \mathrm{~b}$ \\
\hline $\mathrm{F}_{1} 14 \mathrm{X} 5$ & $9.40 \mathrm{f}$ & $3.33 \mathrm{bc}$ & $0.00 \mathrm{~b}$ \\
\hline $\mathrm{F}_{1} 14 \mathrm{X} 8$ & $9.40 \mathrm{f}$ & $0.00 \mathrm{c}$ & $3.33 \mathrm{ab}$ \\
\hline $\mathrm{F}_{1} 14 \mathrm{X} 216$ & $9.40 \mathrm{f}$ & $0.00 \mathrm{c}$ & $3.33 \mathrm{ab}$ \\
\hline $\mathrm{F}_{1} 14 \mathrm{X} 233$ & $8.40 \mathrm{~g}$ & $0.00 \mathrm{c}$ & $0.00 \mathrm{~b}$ \\
\hline $\mathrm{F}_{1} 18 \mathrm{X} 216$ & $13.40 \mathrm{~b}$ & $10.00 \mathrm{ab}$ & $10.00 \mathrm{a}$ \\
\hline $\mathrm{F}_{1} 18 \mathrm{X} 233$ & $10.40 \mathrm{e}$ & $10.00 \mathrm{ab}$ & $0.00 \mathrm{~b}$ \\
\hline $\mathrm{F}_{1} 19 \mathrm{X} 5$ & $8.40 \mathrm{~g}$ & $3.33 \mathrm{bc}$ & $0.00 \mathrm{~b}$ \\
\hline $\mathrm{F}_{1} 19 \mathrm{X} 203$ & $10.40 \mathrm{e}$ & $0.00 \mathrm{c}$ & $0.00 \mathrm{~b}$ \\
\hline $\mathrm{F}_{1} 19 \mathrm{X} 216$ & $8.40 \mathrm{~g}$ & $0.00 \mathrm{c}$ & $0.00 \mathrm{~b}$ \\
\hline$F_{1} 19 X 233$ & $10.40 \mathrm{e}$ & $0.00 \mathrm{c}$ & $3.33 \mathrm{ab}$ \\
\hline $\mathrm{F}_{1} 21 \mathrm{X} 221 \mathrm{~B}$ & $11.40 \mathrm{~d}$ & $6.66 \mathrm{a}-\mathrm{c}$ & $0.00 \mathrm{~b}$ \\
\hline BARI Hybrid Begun 4 & $13.16 \mathrm{~b}$ & $0.00 \mathrm{c}$ & $3.33 \mathrm{ab}$ \\
\hline Level of sig. & $*$ & $*$ & $*$ \\
\hline $\mathrm{CV}(\%)$ & 3.92 & 157.95 & 122.50 \\
\hline
\end{tabular}


The range of fruit infection by EFSB was $7.40-15.40 \%$, while lowest in $\mathrm{F}_{1} 5 \mathrm{X} 8(7.40 \%)$, which was identical to $\mathrm{F}_{1} 13 \mathrm{X} 11(7.45 \%)$ and followed by $\mathrm{F}_{1} 14 \mathrm{X} 233, \mathrm{~F}_{1} 19 \mathrm{X} 5, \mathrm{~F}_{1} 19 \mathrm{X} 216(8.40 \%), \mathrm{F}_{1} 1 \mathrm{X} 216, \mathrm{~F}_{1}$ $14 \mathrm{X} 5, \mathrm{~F}_{1} 14 \mathrm{X} 8, \mathrm{~F}_{1} 14 \mathrm{X} 216(9.40 \%)$ and highest was in $\mathrm{F}_{1} 5 \mathrm{X} 203$ (15.40 \%). In case of bacterial wilt (BW) infestation at field level performance, zero percent incidence was observed in $\mathrm{F}_{1} 1 \mathrm{X} 216, \mathrm{~F}_{1} 1 \mathrm{X} 233, \mathrm{~F}_{1}$ 5X233, $\mathrm{F}_{1}$ 14X8, $\mathrm{F}_{1}$ 14X216, $\mathrm{F}_{1}$ 14X233, $\mathrm{F}_{1}$ 19X203, $\mathrm{F}_{1}$ 19X216, $\mathrm{F}_{1}$ 19X233, BARI Hybrid Begun 4, while maximum was observed in $\mathrm{F}_{1} 5 \mathrm{X} 203(13.33 \%)$. Phomopsis blight diseases zero percent incidence were observed in the lines viz., $\mathrm{F}_{1}$ 1X216, $\mathrm{F}_{1} 1 \mathrm{X} 233, \mathrm{~F}_{1} 4 \mathrm{X} 203, \mathrm{~F}_{1}$ 5X8, $\mathrm{F}_{1}$ 5X203, $\mathrm{F}_{1} 13 \mathrm{X} 11, \mathrm{~F}_{1} 14 \mathrm{X} 5, \mathrm{~F}_{1} 14 \mathrm{X} 233$, $\mathrm{F}_{1}$ 18X233, $\mathrm{F}_{1} 19 \mathrm{X} 5, \mathrm{~F}_{1} 19 \mathrm{X} 203, \mathrm{~F}_{1}$ 19X216, $\mathrm{F}_{1}$ 21X221B. Morphological characteristics of the lines are presented in table 4 .

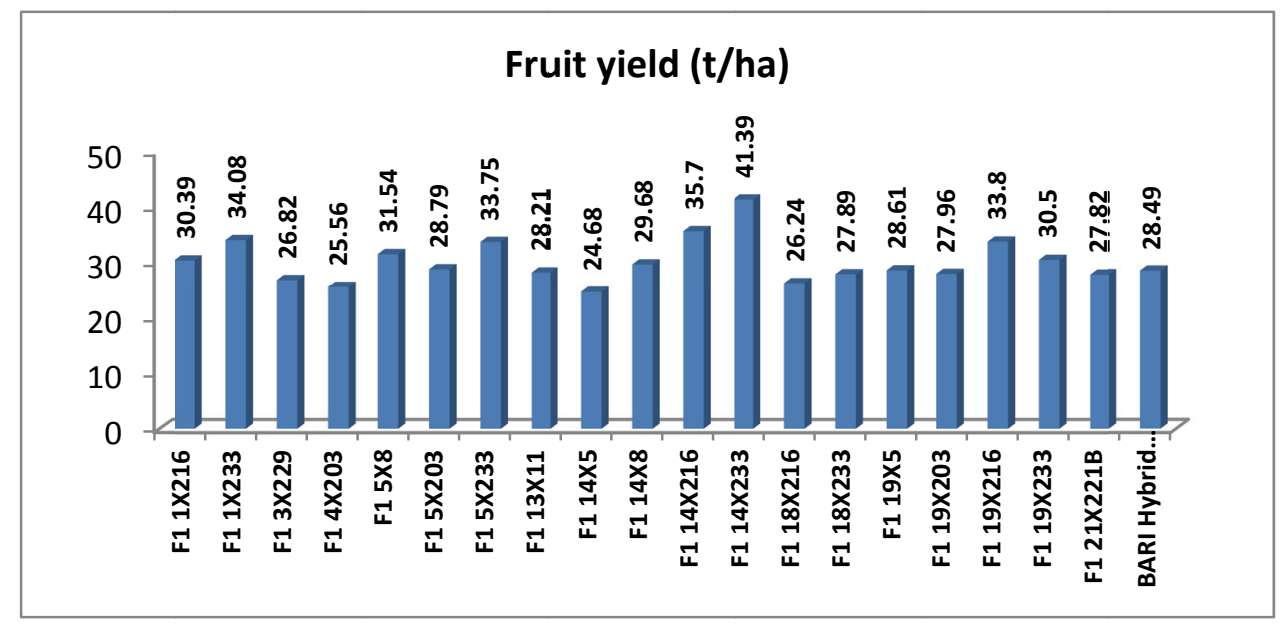

Figure 1. Fruit yield (t/ha) of 20 eggplant hybrids/ variety

Table 5. Fruit shape and colour of 20 eggplant hybrid lines/ variety

\begin{tabular}{|c|c|c|}
\hline Hybrids & Fruit shape & Fruit colour \\
\hline $\mathrm{F}_{1} 1 \mathrm{X} 216$ & Cylindrical & Purple \\
\hline $\mathrm{F}_{1} 1 \mathrm{X} 233$ & Cylindrical & Purple \\
\hline $\mathrm{F}_{1} 3 \mathrm{X} 229$ & Elongate & Light green \\
\hline $\mathrm{F}_{1} 4 \mathrm{X} 203$ & Oblong & Purple \\
\hline $\mathrm{F}_{1} 5 \mathrm{X} 8$ & Elongate & Purple \\
\hline $\mathrm{F}_{1} 5 \mathrm{X} 203$ & Oval & Purple \\
\hline $\mathrm{F}_{1} 5 \mathrm{X} 233$ & Oblong & Purple \\
\hline $\mathrm{F}_{1} 13 \mathrm{X} 11$ & Oval & Light green with white spot at bottom \\
\hline $\mathrm{F}_{1} 14 \mathrm{X} 5$ & Oblong & Purple \\
\hline $\mathrm{F}_{1} 14 \mathrm{X} 8$ & Oblong & Purple \\
\hline $\mathrm{F}_{1} 14 \mathrm{X} 216$ & Elongate & Purple \\
\hline $\mathrm{F}_{1} 14 \mathrm{X} 233$ & Elongate & Purple \\
\hline $\mathrm{F}_{1} 18 \mathrm{X} 216$ & Oblong & Purple \\
\hline $\mathrm{F}_{1} 18 \mathrm{X} 233$ & Oblong & Purple \\
\hline $\mathrm{F}_{1} 19 \times 5$ & Oblong & Purple \\
\hline $\mathrm{F}_{1} 19 \mathrm{X} 203$ & Oval & Purple \\
\hline $\mathrm{F}_{1} 19 \mathrm{X} 216$ & Cylindrical & Purple \\
\hline $\mathrm{F}_{1} 19 \mathrm{X} 233$ & Cylindrical & Purple \\
\hline $\mathrm{F}_{1} 21 \mathrm{X} 221 \mathrm{~B}$ & Oblong & Green with white spot at bottom \\
\hline BARI Hybrid Begun 4 & Oval & Light green \\
\hline
\end{tabular}

The yield range of eggplant hybrids was 24.68- 41.49t/ha. The highest fruit yield was recorded from the line $\mathrm{F}_{1}$ 14X233 (41.39 t/ha), followed by $\mathrm{F}_{1}$ 14X216 (35.70 t/ha), $\mathrm{F}_{1} 1 \mathrm{X} 233(34.08 \mathrm{t} / \mathrm{ha}), \mathrm{F}_{1}$ 19X216 
(33.80 t/ha), $\mathrm{F}_{1} 5 \mathrm{X} 233$ (33.75 t/ha), $\mathrm{F}_{1} 5 \mathrm{X} 8$ (31.54 t/ha), and lowest yield were recorded from $\mathrm{F}_{1} 14 \mathrm{X} 5$ (24.68 t/ha).

Four types of fruit shape were observed among the lines viz., elongate (4 hybrids), cylindrical (4 hybrids), oval (4 hybrids), oblong ( 8 hybrids), while in term of fruit colour, all the hybrids were purple in colour except four hybrids viz., $F_{1} 3$ X229 and BARI Hybrid Begun 4 (Light green), F 13 X11 and $F_{1}$ 21X221B (Green with white spot at bottom).

\section{Conclusion}

Though the yield was higher in the hybrids $\mathrm{F}_{1} 14 \mathrm{X} 233, \mathrm{~F}_{1} 14 \mathrm{X} 216, \mathrm{~F}_{1} 1 \mathrm{X} 233, \mathrm{~F}_{1} 19 \mathrm{X} 216, \mathrm{~F}_{1} 5 \mathrm{X} 233, \mathrm{~F}_{1}$ $5 \mathrm{X} 8$, but considering earliness, tolerance to high temperature, fruit infection by EFSB, bacterial wilt and phomopsis blight infestation, attractive fruit shape and fruit colour, the hybrids $\mathrm{F}_{1} 1 \mathrm{X} 233, \mathrm{~F}_{1}$ 5X8, $\mathrm{F}_{1}$ 13X11, $\mathrm{F}_{1}$ 14X233, $\mathrm{F}_{1}$ 14X216, $\mathrm{F}_{1}$ 19X216, $\mathrm{F}_{1}$ 19X233, $\mathrm{F}_{1} 21 \mathrm{X} 221 \mathrm{~B}$ were found promising. So these seven hybrids can be selected for advance trial.

\section{References}

1. Arup Chattopadhyay, Tania Seth, Subrata Dutta, Partha Pratim Ghosh, Sankhendu Bikash Chattopadhyay, Debasis Majumder. 2012. Breeding Eggplant for Higher Productivity and Bacterial Wilt Tolerance, International Journal of Vegetable Science, 18:376-392, 2012.

2. Ajjappalavara, P.S., P.R. Dharmatti, P.M. Salimath, R.V. Patil, M.S. Patil, and P.U. Krishnaraj. 2008. Genetics of bacterial wilt resistance in brinjal. Karnataka J. Agr. Sci. 21:424-427

3. Bavage, M.S., M.B. Madalageri, and R. Mulge. 2005. Hybrid performance of round fruited brinjal (Solanum melongena L.). Karnataka J. Hort. 1:95-97.

4. Cao, B.-H., J.-J.Lei, W. Yong, and G.-J. Chen. 2009. Inheritance and identification of SCAR marker linked to bacterial wilt-resistance in eggplant. Afr. J. Biotechnol. 8(20):5201-5207.

5. Chaudhary, D.R. 2000.Inheritance of resistance to bacterial wilt (Ralstonia solanacearum E. F. Smith) in eggplant. Haryana J. Hort. Sci. 29(1):89-90.

6. Dharwad, N., S.A. Patil, and P.M. Salimath. 2011. Study on genetic diversity and its relation to heterosis in brinjal (Solanum melongena L.). Karnataka J. Agr. Sci. 24(2):110-113.

7. Feng, L.L., D.Y. Qu, L.P. Jin, and Y. Lian. 2003. Genetic analysis of resistance to bacterial wilt (Ralstonia solanacearum) in eggplant (Solanum melongena L.). Acta Hort. Sinica 30(2):163-166.

8. Gopinath, G. and B.B. Madalageri. 1986. Bacterial wilt (Pseudomonas solanacearum E. F. Smith) resistance in eggplant. Veg. Sci. 13:189-195.

9. Kakikazi, Y. 1931. Hybrid vigour in eggplant and its potential utilization. J. Hered. 21:253-258.

10. Zhu, H., Y.G. Yao, Z.M. Liu, J.G. Yang, and H.M. Chen. 2004. On resistance to bacterial wilt in eggplant (Solanum melongena L.). J. Hunas Agr. Univ. 30(3):288-289. 